\title{
Primary Melanoma of the Vagina Treated by Imatinib: Case Report
}

\author{
Karima Oualla ${ }^{1 *}$, Fatimazahra El'mrabet ${ }^{1}$, Hind Elfatemi ${ }^{2}$, Samia Arifi ${ }^{1}$, Nawfel Mellas ${ }^{1}$, \\ Abdelaziz Banani ${ }^{2}$, Siham Tizniti ${ }^{2}$, Afaf Amarti $^{2}$, Omar Elmesbahi ${ }^{1}$ \\ ${ }^{1}$ Department of Medical Oncology, Hassan II University Hospital, Fez, Morocco; ${ }^{2}$ Department of Pathology, Hassan II University \\ Hospital, Fez, Morocco. \\ Email: "karimarauf@hotmail.com
}

Received June $18^{\text {th }}, 2013$; revised July $20^{\text {th }}, 2013$; accepted July $30^{\text {th }}, 2013$

Copyright (C) 2013 Karima Oualla et al. This is an open access article distributed under the Creative Commons Attribution License, which permits unrestricted use, distribution, and reproduction in any medium, provided the original work is properly cited.

\begin{abstract}
Melanoma of the vagina is a rare lesion. It results from the malignant transformation of ectopic melanocytes occurring in post-menopause. The CKIT is expressed in 36\% of cases in addition to melanoma markers. Prognosis is poor especially for inoperable and disseminated forms despite systemic therapy. Treatment with Imatinib is an option in cases of metastatic mucosal melanoma with CKIT mutation or amplification. We report the case of post-menopausal women, treated at Hassan II University Hospital, diagnosed with metastatic melanoma of the vagina, that didn't respond to first line of chemotherapy, and received Imatinib as second line of treatment, with good clinical response and durable stability at radiological assessment.
\end{abstract}

Keywords: Mucosal Melanoma; Vagina; CKIT; Imatinib

\section{Introduction}

Malignant melanoma of the vagina, a very rare malignancy, has a notoriously aggressive behavior associated with a high risk of local recurrence and distant metastasis. Various treatment options for this disease are available but no standard guidelines, therefore the treatment is still a big challenge. The aim of this work is to show the rarity of this disease, describe the clinical, pathological features and the treatment management, especially with a new targeted therapy that seems very hopeful.

\section{Case Presentation}

We report the case of 70 years old patient, postmenopausal, treated in the Department of Medical Oncology at Hassan II University Hospital. She presented 8 months ago vaginal bleeding. The gynecological examination objectified a vaginal ulcerative burgeoning mass occupying the entire vagina and cervix. A biopsy was performed.

Histology confirmed the diagnosis of melanoma of the vagina, with positive Melan A, HMB-45 and S-100 protein (Figures 1 and 2). CKIT was also positive at the immunohistochemical evaluation (Figure 3). The pelvic scan

${ }^{*}$ Corresponding author. showed a vaginal tumor with important locally extension and locoregional lymph nodes (Figure 4), and secondary lung lesions on the distant staging. The patient received first line chemotherapy based on carboplatin-paclitaxel. The evolution was marked, after 3 cycles, by locally and distant progression. Since the tumor expressed CKIT, the patient received Imatinib as second-line treatment, with improvement of clinical symptoms especially bleeding and long duration of disease stability on radiologic evaluation.

\section{Discussion}

Vaginal malignant melanoma, arisen from melanocytes, which may be present in the epithelium of the vagina in around $3 \%$ of women, is a rare gynecological malignancy. This extremely rare entity is primarily a disease of postmenopausal women. It accounts for less than $1 \%$ of all malignant melanomas, and less than $3 \%$ of all primary malignant tumors of the vagina [1].

Primary vaginal melanoma is less frequent than other melanomas of the female genital tract in both Europe and America [2,3]. In contrast, Ikegaya et al. reported that approximately $52 \%$ of female genital tract melanomas in Japan are primary vaginal melanomas [4]. These differ- 


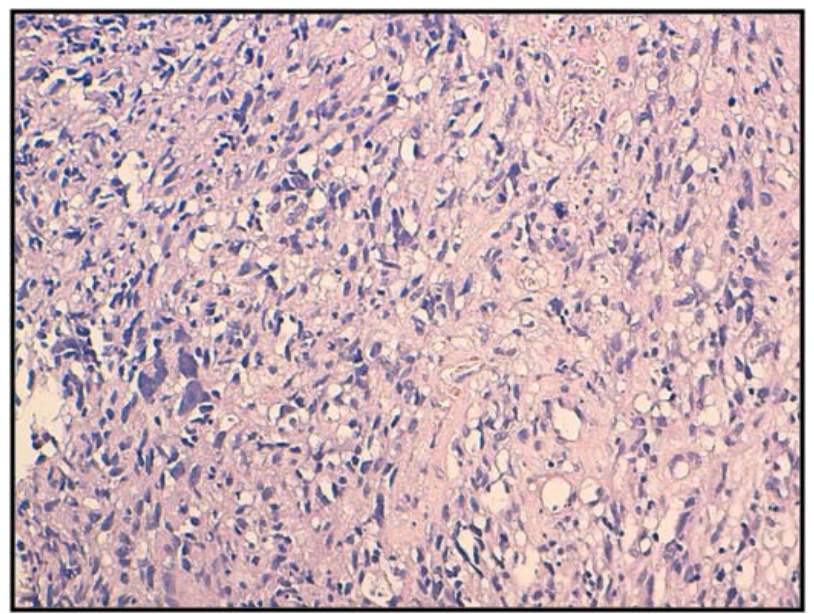

Figure 1. histological appearance of melanoma of the vagina (HES * 20).

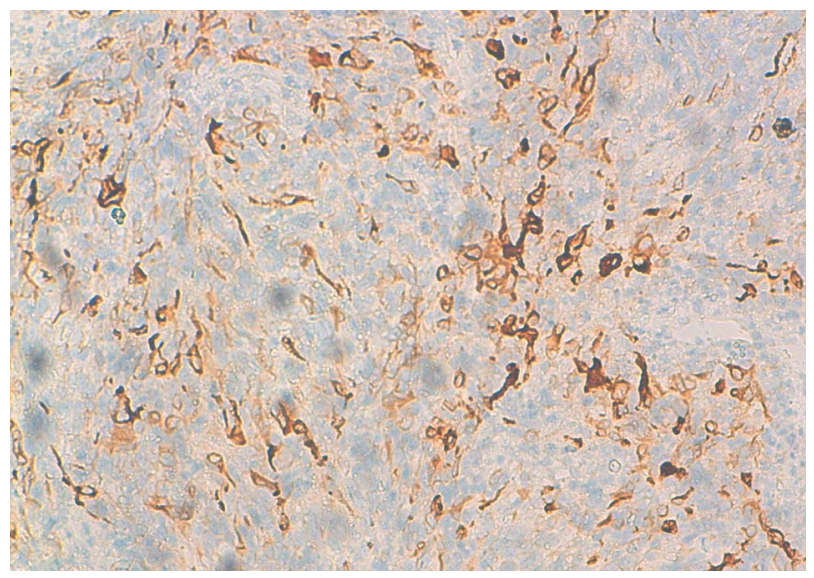

Figure 2. Immunohistochemical analysis with anti-Melan A.

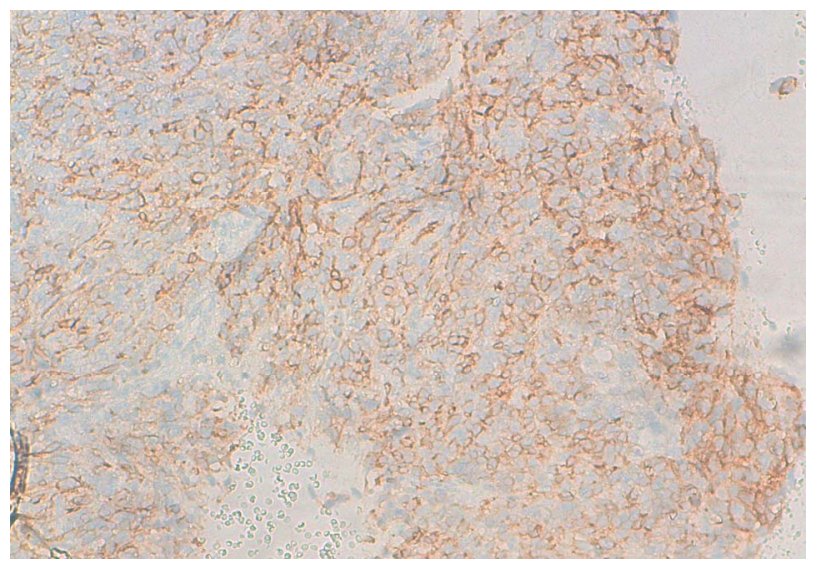

Figure 3. Immunohistochemical analysis with anti-CD117.

ences in incidence seem to be caused by race.

Most vaginal melanomas are diagnosed in the 6th or 7th decade of life in postmenopausal women [5]. The most common symptoms are vaginal bleeding, vaginal discharge and feeling a mass in the vagina. It is mostly

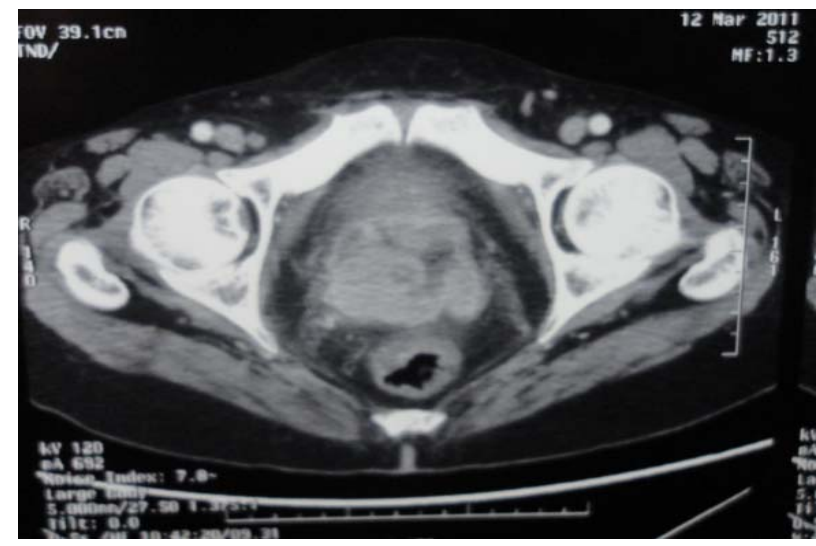

Figure 4. Pelvic scan: vaginal melanoma locally extended.

localized in the anterior wall in lower one third of the vagina [6,7].

The 5-year survival rate for this very aggressive, rapidly growing tumour is very poor, and is reported to be 0 - 25\% irrespective of the chosen therapy [8,9]. Vaginal melanomas are usually only detected in advanced stages. Around $50 \%$ of patients already have lymph node metastasis at diagnosis, and $20 \%$ have distant metastasis

The role of chemotherapy in vaginal melanoma has not been completely defined because of the small number of cases. Dacarbazine has been considered the standard of treatment for metastatic or recurrent melanoma since 1972. Other useful antineoplasm agents include Interleukin-2, temozolomide, platinum analogs, nitrosoureas, and taxanes. The response rate of these single agents is $11 \mathrm{e} 22 \%$, with median overall survival of 5.6 - 11 months.

Radiation therapy also for local control has been mostly offered in the following two conditions, surgically nonresectable disease or as an adjuvant therapy in case of pathologically positive margins or positive lymphadenopathy [2].

Regarding targeted therapies, only Vemurafenib and Ipilimumab had shown their efficacy in metastatic melanoma on phase III trials. But only Ipilimumab has been shown to improve overall survival. For Vemurafenib, approximately $40 \%$ to $60 \%$ of cutaneous melanomas carry mutations in BRAF V600E [10]. But mucosal melanomas have lower frequency of this mutation, comparing to other histological sub-types.

Melanoma is composed of several biologically distinct subtypes, each with unique genetic and clinical features, and each likely to respond differently to any one therapeutic strategy.

Melanoma arising from mucosal infrequently has BRAF mutations, but commonly have amplifications or activating mutations of KIT [11,12].

KIT is a type III transmembrane receptor tyrosine kinase 10. Binding of its ligand, stem cell factor, results in receptor dimerization, autophosphorylation, and activa- 
tion of several signaling pathways; thereby, mediating cancer cell growth, proliferation, invasion, metastasis, and inhibition of apoptosis.

The importance of KIT in normal melanocyte development is well established [13,14]; however, its role as an oncogene and therapeutic target in melanoma has only recently become clear. Although KIT is expressed in some melanomas, loss of expression is observed with progression of disease from superficial to invasive to metastatic stages, suggesting that KIT possesses tumor suppressive functions.

Furthermore, 3 phase 2 studies of metastatic melanoma treated with imatinib mesylate, an orally available ATPcompetitive inhibitor of several tyrosine kinases including KIT, did not demonstrate clinical activity $[15,16]$. These trials accrued before the discovery of activating mutations of KIT in melanoma and did not select patients based on the presence of KIT mutations or amplification.

KIT is an established therapeutic target in cancers with activating mutations of KIT, such as gastrointestinal stromale tumors (GIST), and significant benefit is achieved with various small molecule inhibitors of KIT including imatinib mesylate [17). Several melanoma cell lines with KIT mutations are highly sensitive to imatinib mesylate $[18,19]$. Furthermore, several patients with melanoma harboring KIT alterations, including a K642E mutation as well as a 7-codon duplication of exon 11, have been reported to achieve major durable responses to Imatinib mesylate [20,21].

In our patient, we were able with Imatinib, to improve clinical symptoms especially bleeding, and to keep durable stability of this disease which is at an advanced stage with poor prognosis.

\section{Conclusion}

Melanoma of the vagina is a rare and aggressive cancer. The prognosis is poor for metastatic stage despite the systemic therapy. New developments with targeted therapies are hopeful.

\section{REFERENCES}

[1] G. Borazjoni, K. A. Prem, T. Ogakaki, et al., "Primary malignant Melanoma of the Vagina: A Clinicopathological Analysis of 10 Cases,” Gynecologic Oncology, Vol. 37, No. 2, 1990, pp. 264-267. doi:10.1016/0090-8258(90)90345-L

[2] W. T. Creasman, J. L. Phillips and H. R. Menck, "The National Cancer Data Base Report on Cancer of the Vagina," Cancer, Vol. 83, No. 5, 1998, pp. 1033-1040. doi:10.1002/(SICI)1097-0142(19980901)83:5<1033::AID -CNCR30>3.0.CO;2-6

[3] B. Ragnarsson-Olding, H. Johansson, L. E. Rutqvist, et al., "Malignant Melanoma of the Vulva and Vagina. Trends in Incidence, Age Distribution, and Long-Term
Survival among 245 Consecutive Cases in Sweden 1960 1984," Cancer, Vol. 71, No. 5, 1993, pp. 1893-1897. doi:10.1002/1097-0142(19930301)71:5<1893::AID-CNC $\underline{\mathrm{R} 2820710528>3.0 . \mathrm{CO} ; 2-7}$

[4] H. Ikegaya, T. Iwasaki, I. Matsuda, et al., "Primary Amelanotic Malignant Melanoma of The Vagina-A Case Report,” Japan Journal of Cancer Clinics, Vol. 33, 1987, pp. 1515-1523 (in Japanese).

[5] D. Gupta, A. Malpica, M. T. Deavers, et al., "Vaginal Melanoma: A Clinicopathologic and Immunohistochemical Study of 26 Cases,” American Journal of Surgical Pathology, Vol. 26, No. 11, 2002, pp. 1450-1457. doi:10.1097/00000478-200211000-00007

[6] B. Piura, "Management of Primary Melanoma of the Female Urogenital Tract,” Lancet Oncology, Vol. 9, No. 10, 2008, pp. 973-981. doi:10.1016/S1470-2045(08)70254-7

[7] V. E. Sugiyamaa, J. K. Chana and D. S. Kappb, "Management of Melanomas of the Female Genital Tract," Current Opinion in Oncology, Vol. 20, No. 5, 2008, pp. 565-569. doi:10.1097/CCO.0b013e32830b0dda

[8] E. Petru, F. Nagele, K. Czerwenka, et al., "Primary Malignant Melanoma of the Vagina: A Long Term Remission Following Radiation Therapy," Gynecologic Oncology, Vol. 70, No. 1, 1998, pp. 23-26. doi:10.1006/gyno.1998.4982

[9] M. A. Weinstock, "Malignant Melanoma of the Vulva and Vagina in the United States: Patterns of Incidence and Population-Based Estimates of Survival," American Journal of Obstetrics \& Gynecology, Vol. 171, No. 5, 1994, pp. 1225-1230. doi:10.1016/0002-9378(94)90137-6

[10] H. Davies, G. R. Bignell, C. Cox, et al., "Mutations of the BRAF Gene in Human Cancer," Nature, Vol. 417, No. 6892, 2002, pp. 949-954. doi:10.1038/nature00766

[11] J. A. Curtin, K. Busam, D. Pinkel and B. C. Bastian, “Somatic Activation of KIT in Distinct Subtypes of Melanoma," Journal of Clinical Oncology, Vol. 24, No. 26, 2006, pp. 4340-4346. doi:10.1200/JCO.2006.06.2984

[12] C. Beadling, E. Jacobson-Dunlop, F. S. Hodi, et al., "KIT Gene Mutations and Copy Number in Melanoma Subtypes," Clinical Cancer Research, Vol. 14, No. 21, 2008, pp. 6821-6828. doi:10.1158/1078-0432.CCR-08-0575

[13] L. Hou, J. J. Panthier and H. Arnheiter, "Signaling and Transcriptional Regulation in the Neural Crest-Derived Melanocyte Lineage: Interactions between KIT and MITF,” Development, Vol. 127, No. 24, 2000, pp. 53795389.

[14] V. Alexeev and K. Yoon, "Distinctive Role of the cKit Receptor Tyrosine Kinase Signaling in Mammalian Melanocytes," Journal of Investigative Dermatology, Vol. 126, No. 5, 2006, pp. 1102-1110. doi:10.1038/sj.jid.5700125

[15] K. Wyman, M. B. Atkins, V. Prieto, et al., "Multicenter Phase II Trial of High-Dose Imatinib Mesylate in Metastatic Melanoma: Significant Toxicity with No Clinical Efficacy,” Cancer, Vol. 106, No. 9, 2006, pp. 2005-2011. doi:10.1002/cncr.21834

[16] K. B. Kim, O. Eton, D. W. Davis, et al., "Phase II Trial of Imatinib Mesylate in Patients with Metastatic Mela- 
noma,” British Journal of Cancer, Vol. 99, No. 5, 2008, pp. 734-740. doi:10.1038/sj.bjc.6604482

[17] G. D. Demetri, M. von Mehren, C. D. Blanke, et al., "Efficacy and Safety of Imatinib Mesylate in Advanced Gastrointestinal Stromal Tumors," New England Journal of Medicine, Vol. 347, No. 7, 2002, pp. 472-480. doi:10.1056/NEJMoa020461

[18] C. R. Antonescu, K. J. Busam, T. D. Francone, et al., "L576P KIT Mutation in Anal Melanomas Correlates with KIT Protein Expression and Is Sensitive to Specific Kinase Inhibition,” International Journal of Cancer, Vol. 121, No. 2, 2007, pp. 257-264. doi:10.1002/ijc.22681

[19] X. Jiang, J. Zhou, N. K. Yuen, et al., “Imatinib Targeting of KIT-Mutant Oncoprotein in Melanoma,” Clinical Cancer Research, Vol. 14, No. 23, 2008, pp. 7726-7732. doi:10.1158/1078-0432.CCR-08-1144

[20] F. S. Hodi, P. Friedlander, C. L. Corless, et al., "Major response to Imatinib Mesylate in KIT-Mutated Melanoma,” Journal of Clinical Oncology, Vol. 26, No. 12, 2008, pp. 2046-2051. doi:10.1200/JCO.2007.14.0707

[21] J. Lutzky, J. Bauer and B. C. Bastian, "Dose-Dependent, Complete Response to Imatinib of a Metastatic Mucosal Melanoma with a K642E KIT Mutation,” Pigment Cell \& Melanoma Research, Vol. 21, No. 4, 2008, pp. 492-493. doi:10.1111/j.1755-148X.2008.00475.X 